\title{
Analysis of Magnetization Mechanism for NdFeB Magnet Using Spin-Polarized Scanning Electron Microscopy (Spin SEM)
}

\author{
T. Kohashi, K. Motai, T. Nishiuchi*, T. Maki*, and S. Hirosawa* \\ Central Research Laboratory, Hitachi, Ltd., 1-280, Higashi-koigakubo Kokubunji-shi, Tokyo 185-8601, Japan \\ *NEOMAX Company, Hitachi Metals, Ltd., 2-15-17 Egawa, Shimamoto-cho, Osaka 618-0013, Japan
}

The magnetization process of $\mathrm{Nd}-\mathrm{Fe}-\mathrm{B}$ bonded magnets prepared by the Hydrogenation-Disproportionation-Desorption-Recombination (HDDR) process was studied by observing magnetic domain structures using spin-polarized scanning electron microscopy (spin SEM). The positional relationship between the grain boundaries and the magnetic domain walls is clearly imaged. The result revealed that these magnets contain grains with a multi-domain structure in the thermally demagnetized state. The domain wall motions in grains with multi-domains are dominant in weak magnetic fields in the initial magnetization process, whereas fraction of the grains with a single domain increases with further increasing the magnetic field. The coercivity variations in these grains were also visualized.

Key words: magnetic domain, NdFeB, SEM, magnetization process, HDDR, bonded magnet

\section{Introduction}

Permanent magnets based on the NdFeB compound have exhibited a high energy-product/volume ratio and have been studied intensively to achieve higher performance. Recently, the application of this magnet has been extended to motors used in hybrid cars or battery vehicles, that demand further improvement in the performance of this class of magnets. Among methods of producing this $\mathrm{Nd}-\mathrm{Fe}-\mathrm{B}$ magnet, the Hydrogenation-Disproportionation-Desorption-Recombi nation (HDDR) process is well-known for producing fine powders with high coercivity ${ }^{1,2}$. The size of the fine grains produced by this method is about $0.3 \mu \mathrm{m}$, which is much smaller than that of the sintered $\mathrm{NdFeB}$, and the direction of the anisotropy axis of each grain can be aligned. Therefore, these powders are used in manufacturing bonded permanent magnets, which can be fabricated into a desired shape to be used in compact motors such as those for automobiles and DC-fans. Properties, especially the coercivity, of this bonded magnet are, therefore, also being intensively studied to maintain magnetic stability under high-temperature conditions. For this purpose, microscopic studies on crystalline and magnetic domain structures, which are related to the crucial factor to determine the coercivity, have been reported ${ }^{3-8)}$.

The grain size of the HDDR bonded magnet is close to the critical diameter where one isolated grain of $\mathrm{Nd}_{2} \mathrm{Fe}_{14} \mathrm{~B}$ cannot have a multi-domain structure. Therefore, it is not clear whether the grains of an HDDR bonded magnet have a single domain or multi-domain structure. Magnetic domain observations in a part of the magnetization process of the HDDR magnet using Lorenz microscopy or magnetic force microscopy (MFM) have been reported ${ }^{7,8)}$, and they suggested that this magnet has a multi-domain structure in the thermally demagnetized state, and it has only a single-domain structure after applying a sufficiently strong magnetic field.

Taking these results into account, we observed domain structures in the magnetization process from the thermally demagnetized state to the saturated state, using spin-polarized scanning electron microscopy (spin SEM) ${ }^{9,10)}$. In a spin SEM measurement, the spin polarization of secondary electrons from the ferromagnetic sample is analyzed, and high-resolution domain images are obtained. Moreover, the sample does not need to be a thin film as in the case of Lorenz microscopy. The vector configuration of magnetization on the sample surface is directly imaged in spin-SEM, while the magnetic flux density above the sample surface is visualized in the case of MFM. By using the spin SEM, therefore, we can study this subject from a different point of view than those of previous reports. Particularly, the sample can be in a bulk form and the relation between the magnetization and the grain structures can be visualized more directly. And the coercivity variations may be visualized by observing the whole magnetization process. A set of recoil curves of this bonded magnet is also obtained to understand the magnetization process.

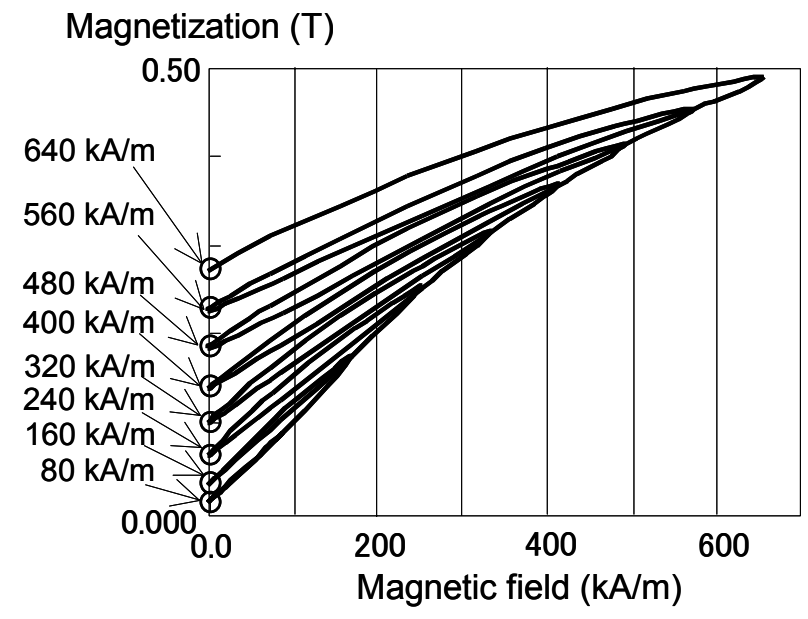

Fig. 1 Recoil curves for $\mathrm{NdFeB}$ bonded magnet. Magnetic field was applied at $640 \mathrm{kA} / \mathrm{m}$ in increments of $80 \mathrm{kA} / \mathrm{m}$. Spin SEM measurements were carried out at remanent magnetization states as indicated by the arrows. 


\section{Recoil Curve Measurement}

First, we mention the recoil curve measurement. We observed remanent states in the spin SEM measurement, which correspond to points of zero fields in a set of recoil curves. It is, therefore, important to measure a recoil curve and compare it to magnetic domain images to analyze the magnetization process ${ }^{11,12)}$. The sample was an anisotropic bonded magnet made of HDDR powder, whose grain diameter and coercive force were $0.3 \mu \mathrm{m}$ and $1040 \mathrm{kA} / \mathrm{m}$, respectively. The initial condition of this sample was a thermally demagnetized state. Then magnetic fields were applied to the sample in the grain-oriented direction, and recoil curves were measured using a vibrating sample magnetometer (VSM). The result is shown in Fig. 1. As the field increases, the magnetization increases following a gradual convex curve. In other words, the curve is rather steep until $400 \mathrm{kA} / \mathrm{m}$, and, after that, its slope becomes modest. On the other hand, remanent magnetization and hysteresis of the magnetization curve are small in the magnetic field smaller than $160 \mathrm{kA} / \mathrm{m}$, while they become larger from $240 \mathrm{kA} / \mathrm{m}$ to $640 \mathrm{kA} / \mathrm{m}$. These observations indicate that magnetization process may change between in the weak field and in the strong field regimes. The features in the magnetic domain structures are, therefore, expected to change during the magnetization process.

\section{Magnetic Domain Observation}

We observed magnetic domain structures corresponding to the remanent states indicated by the arrows in the recoil curve in Fig. 1 in the following procedure. For the magnetic domain observation, we prepared the sample in a thermally demagnetized state as used in the recoil curve measurement. The observation plane, which includes the grain-oriented direction, was polished to a mirror surface. Argon ion milling was performed to carve the grain boundaries before the sample was inserted into the spin SEM chamber to determine the shape of each grain in SEM images. Then, domain images of the thermally demagnetized state were taken. Subsequently, the sample was taken out from the spin SEM chamber, and a magnetic field of $80 \mathrm{kA} / \mathrm{m}$ was applied to it in the grain-oriented direction and the magnetic field was decreased to zero in the VSM. Then, magnetic domain images were observed in the spin SEM system again. We repeated this procedure while increasing the strength of the magnetic field to $640 \mathrm{kA} / \mathrm{m}$ in $80-\mathrm{kA} / \mathrm{m}$ increments.

One of the results is shown in Fig. 2. The grains

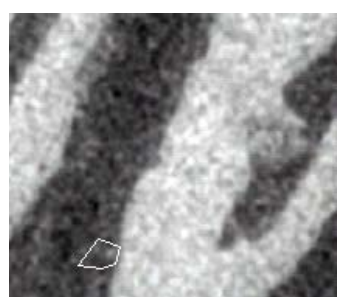

(a)

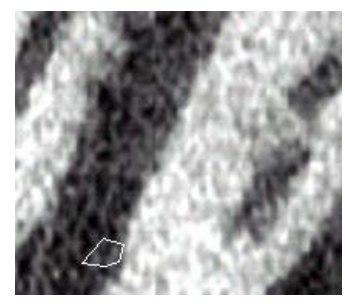

(d)

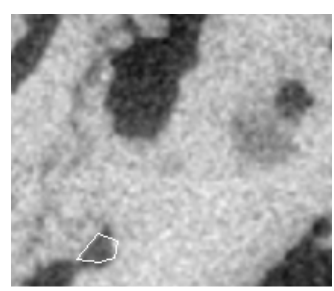

(h)

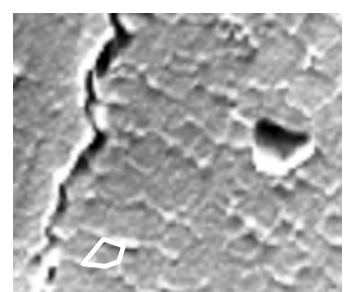

(b)

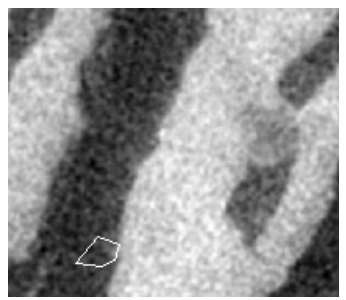

(e)

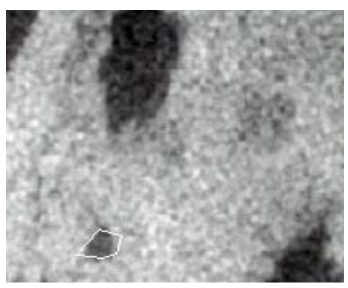

(i)

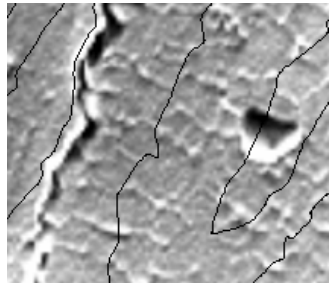

(c)

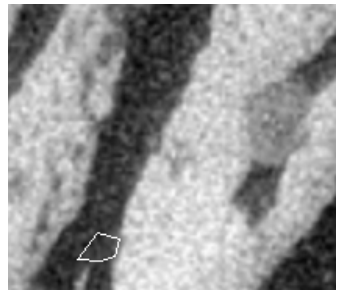

(f)

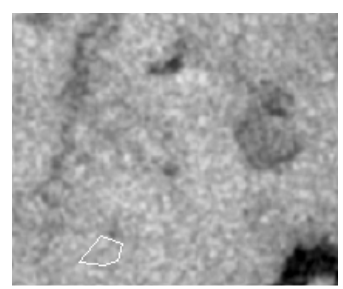

(j)

\section{$1 \mathrm{um}$}

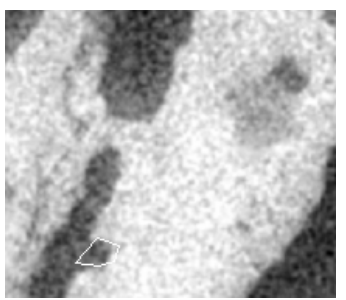

(g)

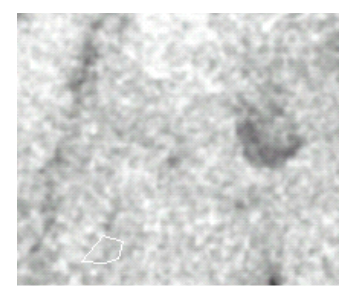

(k)

Fig. 2 Spin SEM images from thermally demagnetized state to remanent magnetization state up to $640 \mathrm{kA} / \mathrm{m}$. (a) magnetic domain image and (b) topography image, and (c) topography image overlapped with the domain wall positions, of the thermally demagnetized state. Magnetic domain images of the remanent magnetization states prepared by the fields of (d) $80 \mathrm{kA} / \mathrm{m}$, (e) $160 \mathrm{kA} / \mathrm{m}$, (f) $240 \mathrm{kA} / \mathrm{m}$, (g) $320 \mathrm{kA} / \mathrm{m}$, (h) $400 \mathrm{kA} / \mathrm{m}$, (i) $480 \mathrm{kA} / \mathrm{m}$, (j) 560 $\mathrm{kA} / \mathrm{m}$ and $(\mathrm{k}) 640 \mathrm{kA} / \mathrm{m}$. Black-and-white contrast in the magnetic domain images indicates vertical magnetization in the image. 
were oriented in the longitudinal direction of the figure. In domain images of the thermally demagnetized state (a), we can see a black-and-white contrast showing the magnetization of the grain-oriented direction. These domains extend in the slightly inclined direction from the longitudinal direction. As shown in Fig. 2 (a) the magnetic domain walls are smooth like straight lines in some areas and have small irregularities in other areas in the thermally demagnetized state. Similar feature of domain structure has been also reported in thermally demagnetized sintered $\mathrm{NdFeB}$ magnets ${ }^{13)}$. In the topography image (b), there is a crack in the left part of the image, and a solid deposit of about $1 \mu \mathrm{m}$ in the right part of the image. Besides those, we can distinguish by weak contrasts small structures of $0.3-0.5 \mu \mathrm{m}$ separately from the magnetic domain structure. These small structures are grains inside the HDDR particles. Fig. 2 (c) shows the combination of (b) with the domain wall position obtained from (a) to study the relationship between the grain shape and the position of the domain wall. The position of the domain walls seem not to be restricted by the grain boundaries, since in some areas they are in the grain boundaries while in other areas they are inside the grain. In the case where the domain walls are inside the grain, those grains have multi-domain structures. This result agrees with former reports ${ }^{7,8)}$.

In Fig. 2 (d)-(k), we show domain structures obtained for the remanent magnetization states prepared under various magnetic fields. Although we had to take out the sample from the spin SEM chamber to apply the magnetic field in the VSM, we took domain images of almost the same area on the sample surface by using the crack and solid deposits in the figure as landmarks. In Fig. 2 (b), we highlighted one of the grains with a white line and its position was indicated in all magnetic

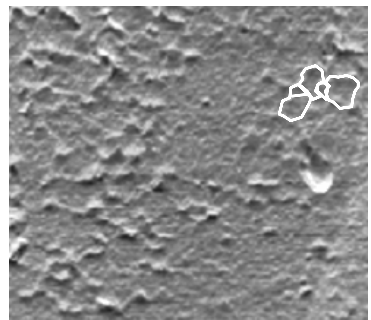

(a)

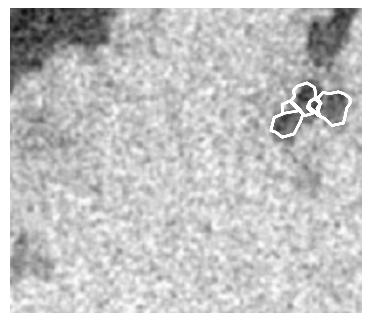

(e)

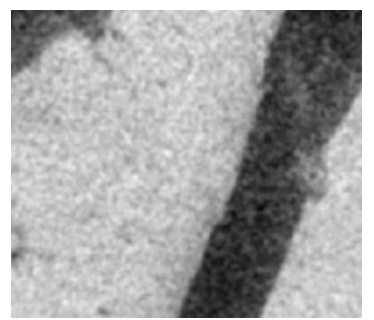

(b)

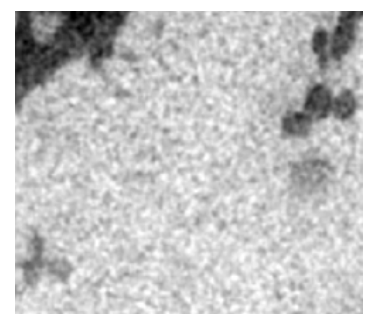

(f)

domain images. There is no difference among magnetic domain shapes in the thermally demagnetized state (a), the remanent magnetization state of $80 \mathrm{kA} / \mathrm{m}$ (d) and that of $160 \mathrm{kA} / \mathrm{m}$ (e). However, the black domains became thinner in the remanent state at $240 \mathrm{kA} / \mathrm{m}$ (f). And the large black domain broke into several domains in the remanent state at $320 \mathrm{kA} / \mathrm{m}(\mathrm{g})$. With further increasing the magnetic field to $560 \mathrm{kA} / \mathrm{m}$, these black domains became smaller, as shown in Fig. 2 (h)-(j). In addition, the magnetic domain wall became ragged as the magnetic field increased. The width of the concave and convex irregularities in the wall was about $0.3-0.5$ $\mu \mathrm{m}$, which is of the same scale as the grain size. This suggests that the magnetic domain walls run along the grain boundaries and each grain has a single domain structure. In fact, the grain indicated by the marker in each figure shows this tendency. In the thermally demagnetized state (a) and the remanent states created by weak magnetizing field up to $240 \mathrm{kA} / \mathrm{m}(\mathrm{d}-\mathrm{f})$, this grain is a part of the large black domain with smooth domain boundaries. In the remanent state following magnetizing at $320 \mathrm{kA} / \mathrm{m}(\mathrm{g})$, the magnetizations near this grain were reversed. Therefore, the boundary of this grain became a part of the magnetic domain wall of the black domain, and this domain wall is no longer as smooth as that in the thermally demagnetized state. A lot more magnetizations were reversed in the range of $400-480$ $\mathrm{kA} / \mathrm{m}$ (h-i), which made this grain a small isolated magnetic domain. Finally, in the remanent state at 560 $\mathrm{kA} / \mathrm{m}(\mathrm{j})$, the magnetization of this grain was reversed.

Spin SEM images of another area of the sample surface are shown in Fig. 3. The topography image is shown in Fig. 3 (a), and the others are magnetic domain images. In the right part of Fig. 3 (b), we can see a black domain expanding in the vertical direction, in which

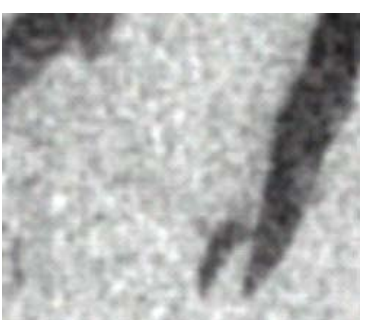

(c)

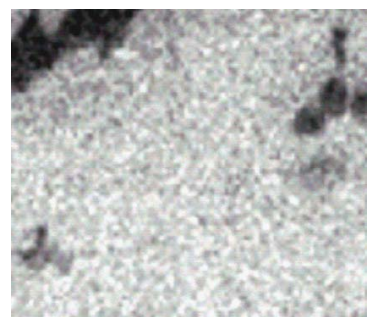

(g)

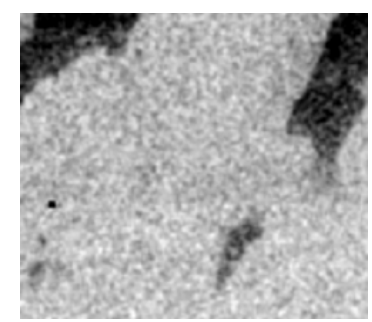

(d)

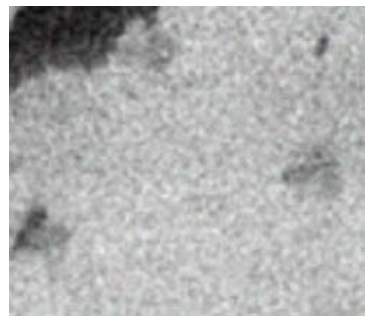

(h)

Fig. 3 Spin SEM images from thermally demagnetized state to remanent magnetization state up to $480 \mathrm{kA} / \mathrm{m}$, obtained in different areas from those in Fig. 2. (a) topography image, and (b) magnetic domain image of the thermally demagnetized state, and magnetic domain images of remanent magnetization states prepared by the fields of (c) $80 \mathrm{kA} / \mathrm{m}$, (d) $160 \mathrm{kA} / \mathrm{m}$, (e) $240 \mathrm{kA} / \mathrm{m}$, (f) $320 \mathrm{kA} / \mathrm{m}$, (g) $400 \mathrm{kA} / \mathrm{m}$, and (h) $480 \mathrm{kA} / \mathrm{m}$. The black and white contrast in the magnetic domain images indicates vertical magnetization in the images. 
domain walls are almost straight lines. This means that the positions of the magnetic domain walls are not affected by grain boundaries. Structures of this domain have already changed from the thermally demagnetized state (b) to the remanent state after magnetizing at 80 $\mathrm{kA} / \mathrm{m}$ (c). The magnetization of a part of the black domain is reversed in (c), where the shape of the domain wall has small irregularities. As the magnetic field increases from $160 \mathrm{kA} / \mathrm{m}$ (d) to $320 \mathrm{kA} / \mathrm{m}$ (f), the black domains become smaller with ragged domain walls. The size of the irregularities of the domain wall is about $0.3-0.5 \mu \mathrm{m}$, which is the same as the size of the grains, suggesting that each grain has a single domain structure. In the domain images of the remanent state at $240-400 \mathrm{kA} / \mathrm{m}(\mathrm{e}-\mathrm{g})$, there are small black domains at the right side of the images (they are marked by the white lines in (e)). We confirmed that corresponding grains for these small magnetic domains can be identified in the topography image (a), which are also marked by white lines. The magnetization of these grains was reversed in $480 \mathrm{kA} / \mathrm{m}$ (h).

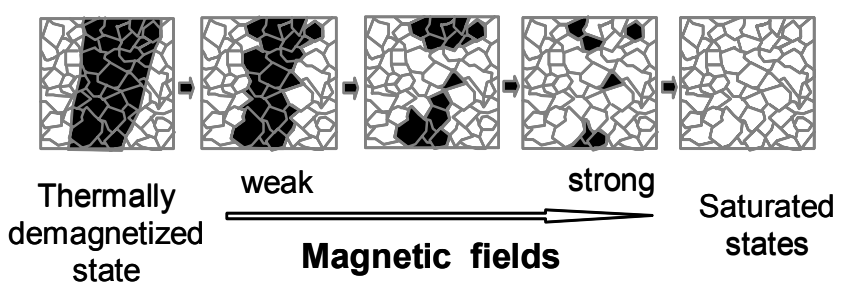

Fig. 4 Schematic of magnetization process from thermally demagnetized state to saturated state, deduced from Figs. 2 and 3.

\section{Discussion}

Figure 4 schematically summarize the characteristics of the magnetization reversal process shown in Figs. 2 and 3. In the thermally demagnetized state, magnetic domain walls are smooth lines. Therefore, they are inside the grains in some cases, leaving these grains in the multi-domain structure. When remanent states were prepared by applying magnetic fields to the sample, the domain walls moved and the magnetic domain shapes changed. In this process of the domain wall motion, the walls sometimes go back to the initial positions after reducing the magnetic field to zero. Therefore, the remanent magnetization does not increase very much and hysteresis is small in recoil curves shown in Fig.1. However, the domain walls were sometimes trapped at the grain boundaries, and the shape of the domain wall become ragged. This means that domain walls have a tendency to stay at the grain boundaries, which leads to a decrease in the number of grains with multi-domains and an increase in the number of those with a single domain. This kind of the magnetization process in permanent magnets was discussed in $\mathrm{NdFeB}$ sintered mangets ${ }^{12-14}$. A further increase in the strength of the magnetic field reverses the magnetization of each grain, and the remaining magnetic domains became small with distorted shapes. After then, such small domains with only a single grain size remain, and finally all magnetization was reversed.
The magnetization in all areas shown in Figs. 2 and 3 were reversed in the remanent state after magnetizing at $640 \mathrm{kA} / \mathrm{m}$. Considering the remanent magnetization shown in the recoil curve in Fig. 1, we surmise that the magnetization reversal occurs in weaker fields in these two areas rather than in other areas of this magnet. This is because the spin SEM images are produced from the magnetization on the sample surface, where the coercivity is weaker than that inside the sample ${ }^{11)}$.

\section{Conclusion}

Our spin SEM image observations have revealed clearly the magnetization process of the anisotropic $\mathrm{NdFeB}$ bonded magnet, and the relation between the grain boundaries and the magnetic domain shapes. It was directly imaged that the domain walls motion in the grain with multi-domains is dominant in the weaker field, and that the magnetization reversal in the grain with a single domain is dominant in stronger fields. This tendency is shown more clearly in this study than in the previous reports ${ }^{7,8}$. Because we observed the magnetization of the bulk sample, not a thin film as in the case of Lorenz microscopy. And we visualized the magnetization itself, while in MFM measurement it is not easy to obtain magnetizations, especially in-plane component in the grains of various shapes as in the case of this study. Adding to this, the whole magnetization process was visualized from the thermally demagnetized state to the saturated state. Moreover, we can see the variation in the coercivity force of each grain, in the remanent state created by strong magnetizing fields. It is important to improve magnetic characteristics of grains whose magnetization is reversed in weaker magnetic fields.

Acknowledgements The authors thank Susumu Soeya of Hitachi, Ltd., for his corporation of preparing the remanent magnetization state of $\mathrm{NdFeB}$ bonded magnet.

\section{References}

1) T. Takeshita, R. Nakayama: Proceedings of the 10th International Workshop on Rare Earth Magnets and their Applications, Kyoto, 1, 551(1989)

2) R. Nakayama and T. Takeshita: J. Alloys and Compounds, 193, 259 (1993).

3) S. Hirosawa, M. Uehara, S. Mino, N. Ishigaki, and T. Tomida: J. Appl. Phys., 81, 4821(1997)

4) Y. Ming, L. Xubo, Xiaoyaofu and Z. Jiuxing: J. Magn. Magn. Mater., 269, 227(2004)

5) J. D. Livingston: J. Appl. Phys., 57, 4137(1985).

6) M. Uehara, T. Tomida, H. Tomizawa, S. Hirosawa and Y. Maehara: J. Magn. Magn. Mater., 159, L304(1996).

7) P. Thompson, O. Gutfleisch, J. N. Chapman and I. R. Harris: J. Phys. D, 30, 1854(1997).

8) T. Maki and S. Hirosawa: J. Magn. Soc. Jpn(in Japanese), 32, 265(2008)

9) H. Matsuyama and K. Koike: J. Electron Microsc. 43,157 (1994).

10) T. Kohashi and K. Motai: AMTC Letters 1, 70(2008).

11) K. Kobayashi, K. Ito, D. Shimizu and K. Hayakawa: $J$. Magn. Soc. Jpn., 31, 393(2007)

12) K. Kobayashi, S Sakamoto, T. Matsushita, K. Hayakawa, and M. Sagawa: Proc. of the $20^{\text {th }}$ International Workshop on Rare Earth Magnet and Their Applications, Crete, 138(2008). 
13) S. Yamamoto, M. Yoneyama, T. Wakita, K. Fukumoto, T. Nakamura, T. Kinoshita, Y. Watanabe, F. Z. Guo, M. Sato, T. Terai and T. Kakeshita: Materials Transaction 49, 2354(2008)
14) K. Kobayashi and D. Givord: J. Magn. Soc. Jpn. 21, 1175 (1997).

Received Nov. 27, 2008; Accepted May 19, 2009. 